\title{
On the Split Personality of Penultimate Proline
}

\author{
Matthew S. Glover, ${ }^{1}$ Liuqing Shi, ${ }^{1}$ Daniel R. Fuller, ${ }^{1}$ Randy J. Arnold, ${ }^{1,3}$ Predrag Radivojac, ${ }^{2}$ \\ David E. Clemmer ${ }^{1}$ \\ ${ }^{1}$ Department of Chemistry, Indiana University, Bloomington, IN 47405, USA \\ ${ }^{2}$ Department of Computer Science and Informatics, Indiana University, Bloomington, IN 47405, USA \\ ${ }^{3}$ Present Address: AB SCIEX, Vaughan, ON L4K 4V8, Canada
}

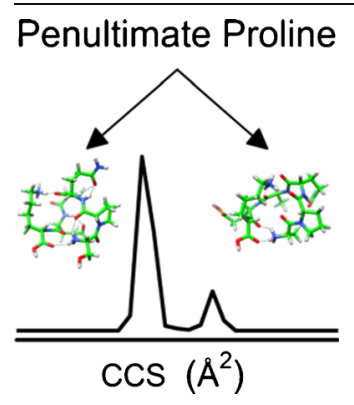

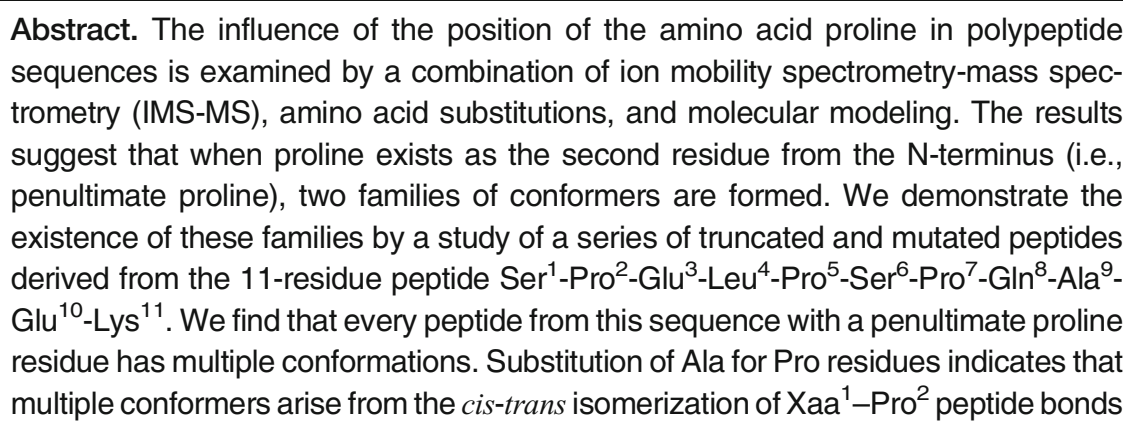
as Xaa-Ala peptide bonds are unlikely to adopt the $c i s$ isomer, and examination of spectra from a library of 58 peptides indicates that $\sim 80 \%$ of sequences show this effect. A simple mechanism suggesting that the barrier between the cis- and trans-proline forms is lowered because of low steric impedance is proposed. This observation may have interesting biological implications as well, and we note that a number of biologically active peptides have penultimate proline residues.

Keywords: Peptide conformation, Penultimate proline, Proline Cis-Trans isomerization, Ion mobility spectrometry

Received: 1 October 2014/Revised: 3 November 2014/Accepted: 10 November 2014/Published Online: 12 December 2014

\section{Introduction}

$\mathrm{W}$ hile examining the influence of the position of different amino acid residues in polypeptide sequences on peptide conformation, we find evidence that proline in the second position from the amino-terminus (i.e., penultimate proline) gives rise to two types of peptide families. This penultimate proline has a propensity to induce two types of stable conformations, having mostly similar populations. Prior work has noted the existence of a penultimate proline in a large number of similarly sized signaling peptides [1-4]. It has been proposed that proline in this position protects the small amino acid chain against further enzymatic degradation [1-3]. The evidence presented here that two populations of conformers are favored suggests that penultimate proline may play another role involving multiple structures that may influence activity and binding.

Electronic supplementary material The online version of this article (doi:10.1007/s13361-014-1049-y) contains supplementary material, which is available to authorized users.

Correspondence to: David E. Clemmer; e-mail: Clemmer@Indiana.edu
Cis-trans isomerization of peptide bonds is one mechanism that can lead to large changes in the overall structure of peptides and proteins [5-8]. Such isomers are most commonly associated with proline, primarily because of the 20 natural amino acids proline is unique in that it has a secondary amine at the backbone nitrogen atom $[9,10]$. This makes it possible for proline to adopt the cis isomer of Xaa-Pro peptide bonds (where Xaa is any amino acid) at a higher frequency than Xaa-Xnp peptide bonds (where $\mathrm{Xnp}$ is any non-proline amino acid) [9, 10]. Although most previous studies classify proline residues either as cis or trans configurations, an increasing number of systems appear to adopt structures that utilize both the cis and trans isomers, and the two forms may have different functions [11]. Thus, it is important to understand the fundamental factors that influence the ability of Xaa-Pro bonds to adopt both cis and trans isomers.

In this study, we use a combination of electrospray ionization (ESI) [12], ion mobility spectrometry (IMS) [13-17], and mass spectrometry (MS) to examine the conformation of a range of peptide sequences. The mobility of an ion through a buffer gas depends upon the shape of the ion, and thus is an effective means of delineating large differences in conformation. When combined with ESI and MS, it is possible to sample 
different conformer populations directly from solution [18-25]. Because of the ability to separate conformations that arise from both cis and trans configurations, IMS-MS is an appealing technique to analyze cis-trans isomerization of prolinecontaining peptides. Previous IMS-MS studies have established the important role that proline plays in the conformations of polypeptide ions [26-29]. Pierson et al. have shown by IMS-MS that proline residues are important in establishing multiple conformations of the nonapeptide bradykinin [27].

In this paper, we primarily examine the influence of proline position on the distribution of conformations observed by IMSMS for the peptide $\mathrm{Ser}^{1}-\mathrm{Pro}^{2}-\mathrm{Glu}^{3}-\mathrm{Leu}^{4}-\mathrm{Pro}^{5}-\mathrm{Ser}^{6}-\mathrm{Pro}^{7}-\mathrm{Gln}^{8}$ $\mathrm{Ala}^{9}-\mathrm{Glu}^{10}$-Lys ${ }^{11}$ - a tryptic peptide found in several proteins across different organisms. For example, it is located at residues 271-281 in the CLPB Protein in Homo sapiens [30]. We are interested in studying this peptide in detail because it has multiple peaks in the ion mobility distribution of the [SPELPSPQAEK + $2 \mathrm{H}]^{2+}$ ion. Examination of this peptide (and related shorter sequences formed as by-products of the synthesis) motivated us to analyze a library of doubly charged peptides, which was available in our laboratory; these studies show that $\sim 80 \%$ of all sequences that contain a penultimate proline adopt multiple conformers. Thus, it appears to be a common feature, in at least some families of sequences. We propose a simple mechanism in which the barrier of the system is lower because of decreased steric impedance associated with the penultimate position to explain the results. Overall, these findings expand upon previous studies $[26,27]$ and help to illuminate the more complex role that proline plays in establishing peptide conformations.

\section{Experimental}

\section{Ion Mobility Spectrometry-Mass Spectrometry}

IMS-MS experiments were performed on a home-built, $\sim 2$-meter instrument described in detail previously [31, 32]. Peptides were electrosprayed from 49.5:49.5:1.0 water:acetonitrile:formic acid solutions. Ions were produced by electrospray ionization with a Triversa Nanomate (Advion Bioscience, Inc., Ithaca, NY, USA). Ions are stored in a Smith-geometry hourglass-shaped ion funnel [33]. Packets of ions are periodically pulsed into the drift tube, which is operated with $3.00 \pm 0.02$ Torr He buffer gas and an electric field of $10 \mathrm{~V} \cdot \mathrm{cm}^{-1}$. Ions exit the drift tube through a differentially pumped region and are analyzed by an orthogonal geometry two-stage reflectron time-of-flight mass spectrometer in a nested fashion [34].

\section{Calculating Collision Cross Sections}

Drift time $\left(t_{D}\right)$ distributions can be converted to collision cross section $(\Omega)$ distributions according to [35],

$\Omega=\frac{(18 \pi)^{1 / 2}}{16} \frac{z e}{\left(k_{b} T\right)^{1 / 2}}\left[\frac{1}{M_{I}}+\frac{1}{M_{B}}\right]^{1 / 2} \frac{t_{D} E}{L} \frac{760}{P} \frac{T}{273.2} \frac{1}{N}$, where $z e$ is the charge of the ion. $K_{b}$ is Boltzmann's constant. $T$ and $P$ are the temperature and pressure, respectively. $E$ and $L$ are the electric field and length of the drift tube, respectively. $\mathrm{N}$ is the neutral number density at STP. For the instrument used in this study, collision cross sections can be calculated two ways. First, we can measure the time it takes the ions to traverse the first region of the drift tube from the source gate to the selection gate at the middle funnel and use Equation 1 directly. Alternatively, we can use the time required for ions to traverse the entire drift tube. However, the ion funnels are not operated with a uniform electric field. Thus, we must calibrate measurements to well-studied systems such as bradykinin. Cross section values obtained from both methods typically agree within $1 \%$. This allows for the conversion of drift time distributions to accurate collision cross section distributions.

In order to compare collision cross section distributions of peptides in which Ala residues have been substituted for Pro residues, we need to account for the intrinsic size difference between the side chains. Previous efforts have focused on developing intrinsic size parameters (ISPs) to provide insight into the average contribution each amino acid residue has to the collision cross section of peptide ions [36-38]. From this work, it was determined that the substitution of Ala for Pro residues leads to peptides with smaller cross sections on average. To account for this intrinsic difference in size, we have shifted our collision cross section distributions by $3.5 \AA^{2}$ for each Pro $\rightarrow$ Ala substitution. This shift in cross section is in good agreement with work by Pierson et al. in which triply charged bradykinin distributions were shifted by $2.5 \AA^{2}$ for each Ala substitution. It is worth mentioning that previous work suggests ISPs are dependent on charge state [36-38]. Furthermore, the $3.5 \AA^{2}$ correction per Pro $\rightarrow$ Ala substitution yields more precisely aligned distributions for the peptides analyzed in this study and both values lead to the same cis and trans isomer assignments.

\section{Peptide Synthesis}

Peptides were synthesized by Fmoc solid-phase synthesis on an Apex 396 peptide synthesizer (AAPPTec, Louisville, KY, USA) similar to the method described previously [27]. Fmoc side-chain protected amino acids and Wang-type polystyrene resins were used (Midwest Biotech, Fischers, IN, USA). Twenty percent piperidine in dimethylformamide was used for $\mathrm{N}^{\alpha}$ deprotections. 1,3-Diisopropylcarbodiimide/6-chloro1-hydroxybenzotriazole or 3-(diethoxyphosphoryloxy)-1,2,3benzotriazin-4(3H)-one was used as the coupling reagent. Peptides were cleaved from the resin with a trifluoroacetic acid:triisopropylsilane:methanol solution at a 18:1:1 ratio, and 5\% 2,2'-(ethylenedioxy)diethanethiol was added for methionine-containing peptides. Peptides were washed and precipitated in diethyl ether. Precipitated products were dried down with a vacuum manifold before reconstituting in $\mathrm{H}_{2} \mathrm{O}$ and finally the ESI solvent. 


\section{Molecular Modeling}

Molecular modeling simulations were completed by a simulated annealing procedure with the Insight II software package (Accelrys, Inc., San Diego, CA, USA) similar to simulations described previously [26]. Simulations were performed on the $[\mathrm{SPQAEK}+2 \mathrm{H}]^{2+}$ ion with charges assigned to the N-terminus and the Lys side chain. Simulated annealing was completed on two initial starting structures that differ by geometry of the $\mathrm{Ser}^{1}-\mathrm{Pro}^{2}$ peptide bond (cis or trans). Ions were heated from 298 to $500 \mathrm{~K}$ over $5 \mathrm{ps}$, equilibrated at $500 \mathrm{~K}$ for $10 \mathrm{ps}$, cooled to $298 \mathrm{~K}$ over $5 \mathrm{ps}$, and equilibrated at $298 \mathrm{~K}$ for $30 \mathrm{ps}$. The extensible systematic force field (ESFF) and a dielectric of 1 were used; 100 trial geometries were obtained from each run. Collision cross sections were calculated using the trajectory method in Mobcal [39].

\section{Results and Discussion}

\section{IMS-MS of SPELPSPQAEK and Truncated Peptides}

Figure 1 shows the two-dimensional IMS-MS plot of ions produced by ESI of the crude product from the synthesis of the peptide SPELPSPQAEK. In addition to the $[\text { SPELPSPQAEK }+2 \mathrm{H}]^{2+}$ ion, we observe the truncated series of peptides (e.g., [PELPSPQAEK $+2 \mathrm{H}]^{2+}$ ) derived from the full-length sequence in relatively high abundance. Truncated peptide sequences result from the inefficiency of peptide synthesis. Although inefficient synthesis is generally thought to be problematic, this makes it possible to obtain IMS-MS measurements for many unique peptide sequences from a single synthesis. From this series of truncated peptides, we observed an interesting trend - as single amino acid residues are added from the 5 residue peptide to the full-length sequence, the distribution of conformers for each peptide is markedly different. That is, some peptides have multiple conformers and others do not. Upon this discovery, we synthesized each individual truncated peptide derived from the SPELPSPQAEK separately to probe this observation in detail. It is worth mentioning that distributions obtained from the two different syntheses are nearly identical.

Figure 2 shows the collision cross section distributions for the peptide [SPELPSPQAEK $+2 \mathrm{H}]^{2+}$ ion and truncated peptides. The distribution has two peaks at 252 and $258 \AA^{2}$ with normalized abundances of $23 \%$ and $77 \%$, respectively. Based on previous studies of proline-containing peptides by ion mobility, one likely explanation for the multiple conformations is that one of the Xaa-Pro bonds adopts both the cis and trans isomer $[26,27]$. However, it is difficult to assign what residue or region of residues in the peptide sequence are important to observing multiple conformer families from the cross section distribution alone. Thus, we will consider the truncated versions of the peptide to examine how the distributions of ions change as a function of length and amino acid composition.

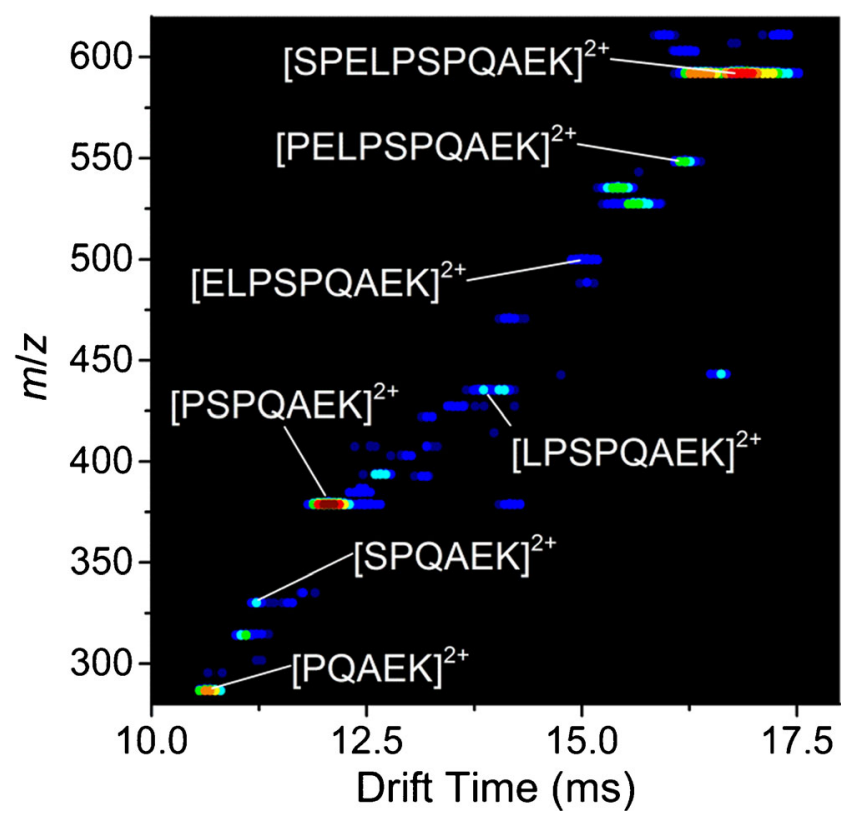

Figure 1. Two-dimensional IMS-MS plot of crude synthesis product of the peptide SPELPSPQAEK electrosprayed from 49.5:49.5:1 water:acetonitrile:formic acid. Doubly charged $[\mathrm{M}+2 \mathrm{H}]^{2+}$ truncated sequences of the fulllength peptide are labeled

Collision cross section distributions for the truncated peptides [PELPSPQAEK $+2 \mathrm{H}]^{2+}$, [ELPSPQAEK $\left.+2 \mathrm{H}\right]^{2+}$, $[\text { LPSPQAEK }+2 \mathrm{H}]^{2+}$, $[\text { PSPQAEK }+2 \mathrm{H}]^{2+}$, [SPQAEK + $2 \mathrm{H}]^{2+}$, and $[\mathrm{PQAEK}+2 \mathrm{H}]^{2+}$ are displayed in Figure 2. It is apparent that the removal of a single amino acid from the $\mathrm{N}-$ terminus dramatically influences the distribution of conformers. The distribution of [PELPSPQAEK $+2 \mathrm{H}]^{2+}$ is markedly different than the full-length sequence. Although both features contain multiple peaks, the minor feature for the $[\text { PELPSPQAEK }+2 \mathrm{H}]^{2+}$ ion is less than $1 \%$ normalized abundance with the major feature at $249 \AA^{2}$. This trend of observing different distributions for each subsequent truncation is observed across all sequences. The distribution for the $[\text { ELPSPQAEK }+2 \mathrm{H}]^{2+}$ ion is dominated by a single peak at $231 \AA^{2}$. The removal of the Glu residue from the N-terminus leads to two highly abundant peaks for the [LPSPQAEK + $2 \mathrm{H}]^{2+}$ ions at 213 and $217 \AA^{2}$. Once again, the removal of the $\mathrm{N}$-terminal amino acid changes the distribution as only a single peak at $185 \AA^{2}$ is observed for the [PSPQAEK $\left.+2 \mathrm{H}\right]^{2+}$ ion. The distribution for the $[\mathrm{SPQAEK}+2 \mathrm{H}]^{2+}$ ion has two features located at 172 and $178 \AA^{2}$. Finally, the $[\mathrm{PQAEK}+2 \mathrm{H}]^{2+}$ ion has a single peak at $164 \AA^{2}$.

It is interesting that we do not observe a specific length at which all subsequent peptides have similar conformer distributions for the sequences analyzed in this study. This suggests that each amino acid residue influences the conformation, and small modifications such as the addition of a single amino acid to the N-terminus can lead to global conformational changes. Distributions oscillate between being dominated by a single peak and multiple peaks as the sequence is extended. The 


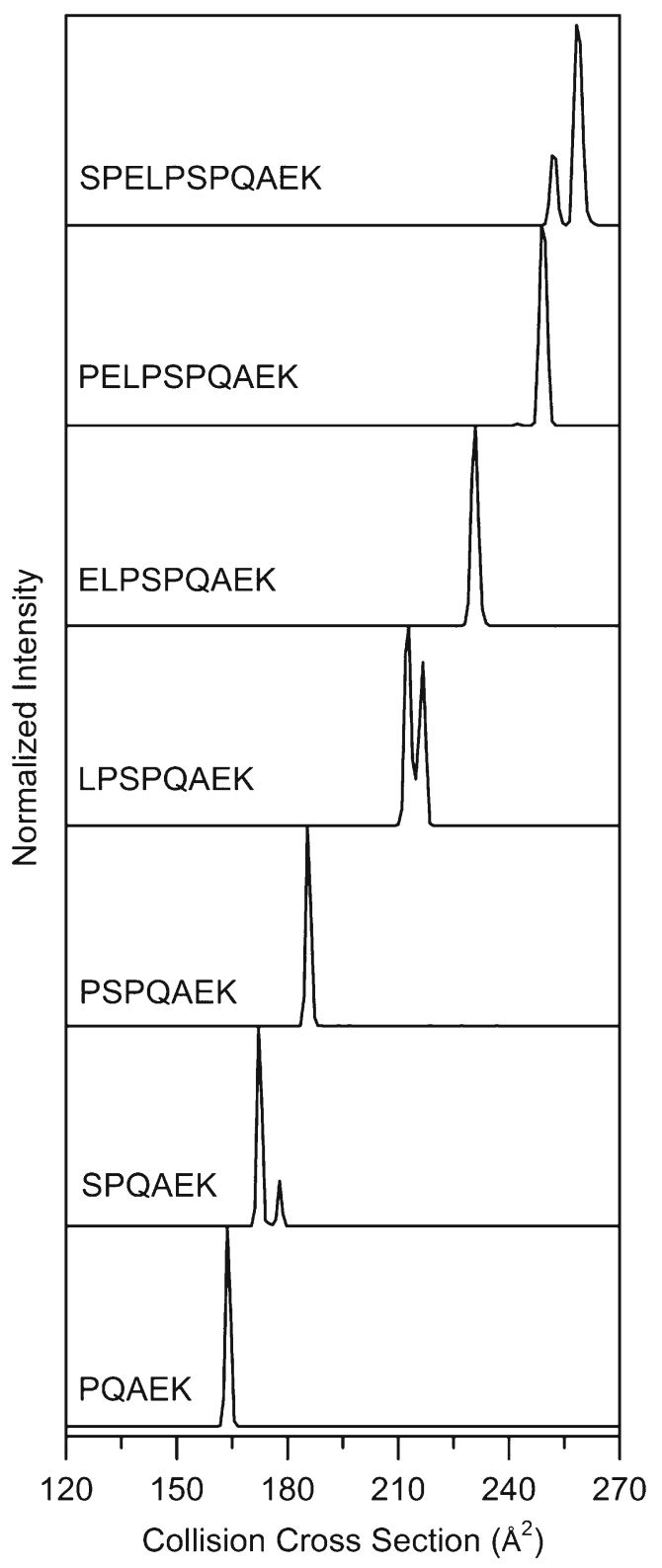

Figure 2. Collision cross section distributions for doubly charged peptide $[\mathrm{M}+2 \mathrm{H}]^{2+}$ ions (where $\mathrm{M}=$ SPELPSPQAEK, PELPSPQAEK, ELPSPQAEK, LPSPQAEK, PSPQAEK, SPQAEK, and PQAEK). Distributions were obtained from IMSMS measurements by integrating the drift bins for a narrow $\mathrm{m} / \mathrm{z}$ window for each ion of interest

$[\text { SPELPSPQAEK }+2 \mathrm{H}]^{2+}$, [LPSPQAEK $\left.+2 \mathrm{H}\right]^{2+}$, and $[\mathrm{SPQAEK}+2 \mathrm{H}]^{2+}$ ion distributions have multiple conformations observed in relatively high abundance, whereas the $[\text { PELPSPQAEK }+2 \mathrm{H}]^{2+},[\text { ELPSPQAEK }+2 \mathrm{H}]^{2+},[$ PSPQAEK $+2 \mathrm{H}]^{2+}$, and $[\mathrm{PQAEK}+2 \mathrm{H}]^{2+}$ ion distributions are dominated by a single peak.

One commonality between the sequences with multiple features, [SPELPSPQAEK $+2 \mathrm{H}]^{2+}$, [LPSPQAEK $\left.+2 \mathrm{H}\right]^{2+}$, and $[\mathrm{SPQAEK}+2 \mathrm{H}]^{2+}$, is the presence of a Pro ${ }^{2}$ residue. However, when the N-terminal residue is removed such that Pro is at position 1 , the distribution is dominated by a single peak. This suggests that the cis-trans isomerization of $\mathrm{Xnp}^{1}$ $\mathrm{Pro}^{2}$ peptide bonds allows for multiple conformer families and not the more centrally positioned residues. It is important to point out that the cis-trans isomerization of proline occurs at the peptide bond preceding proline (i.e., Xaa-Pro). Therefore, $\mathrm{Pro}^{1}$ residues are unable to adopt both cis and trans isomers and, consequently, multiple conformers.

\section{IMS-MS Analysis of Pro Substituted Peptides}

The analysis of the truncated peptides has shown that three sequences, $[\text { SPELPSPQAEK }+2 \mathrm{H}]^{2+}$, $[\text { LPSPQAEK }+2 \mathrm{H}]^{2+}$, and $[\mathrm{SPQAEK}+2 \mathrm{H}]^{2+}$, have multiple conformer families at relatively high abundance. Thus, we will focus on determining the amino acid residues that are important to stabilizing multiple conformations for these sequences. This work was inspired by previous IMS-MS studies by Pierson et al. in which Ala residues were substituted for Pro residues in the nonapeptide bradykinin (BK) [27]. Substituting Ala for Pro residues likely fixes the Xaa-Ala peptide bond in the trans isomer. It was shown that the cis-trans isomerization of proline residues are important for triply charged $[\mathrm{BK}+3 \mathrm{H}]^{3+}$ ion conformations and specific Xaa-Pro bonds were assigned as cis or trans for different conformations.

\section{SPELPSPQAEK Pro $\rightarrow$ Ala Substitutions}

Figure 3 shows the collision cross section distributions for the Pro $\rightarrow$ Ala analogues of the [SPELPSPQAEK $+2 \mathrm{H}]^{2+}$ ions. Throughout this manuscript, cross section distributions for Pro $\rightarrow$ Ala substituted peptides have been corrected for the size difference between proline and alanine as explained above. The cross section distribution for the $\mathrm{Pro}^{2} \rightarrow$ Ala substitution is dominated by a single peak at $252 \AA^{2}$. This suggests that the cis-trans isomerization of $\mathrm{Ser}^{1}-\mathrm{Pro}^{2}$ peptide bond of $[\text { SPELPSPQAEK }+2 \mathrm{H}]^{2+}$ ion is crucial for the conformation at $258 \AA^{2}$. Furthermore, it supports the results from the truncated series that suggest $\operatorname{Pro}^{2}$ residues are important for multiple conformers.

In order to verify that the N-terminal region of the peptide leads to multiple structures, we have analyzed the $\operatorname{Pro}^{5}$ and Pro $^{7}$ substituted peptides. Both [SPELASPQAEK $+2 \mathrm{H}]^{2+}$ and $[\text { SPELPSAQAEK }+2 \mathrm{H}]^{2+}$ distributions have multiple peaks (Figure 3). The collision cross sections of the major peak for both the $\mathrm{Ala}^{5}$ and $\mathrm{Ala}^{7}$ peptides is $260 \AA^{2}$. The conformers at $260 \AA^{2}$ are less than $1 \%$ larger than the major peak at $258 \AA^{2}$ for the peptide [SPELPSPQAEK $+2 \mathrm{H}]^{2+}$ ion. Although we observe a peak near $258 \AA^{2}$ when both $\mathrm{Pro}^{5}$ and $\mathrm{Pro}^{7}$ are substituted, this peak is not observed for the $\mathrm{Pro}^{2}$ substitution. Therefore, we assign the conformation at $258 \AA^{2}$ as cis-Pro ${ }^{2}$, trans-Pro ${ }^{5}$, trans-Pro ${ }^{7}$. A summary of cis-trans isomer assignments and populations can be found in Table 1.tgroup

As mentioned above, both the distributions for the $\mathrm{Pro}^{5} \rightarrow$ Ala and $\mathrm{Pro}^{7} \rightarrow$ Ala sequences have minor features. Although our data suggests that the $\operatorname{Pro}^{5}$ and Pro $^{7}$ residues are not the sites key to having multiple peaks, they dramatically influence the distribution of conformations. The minor features for the 


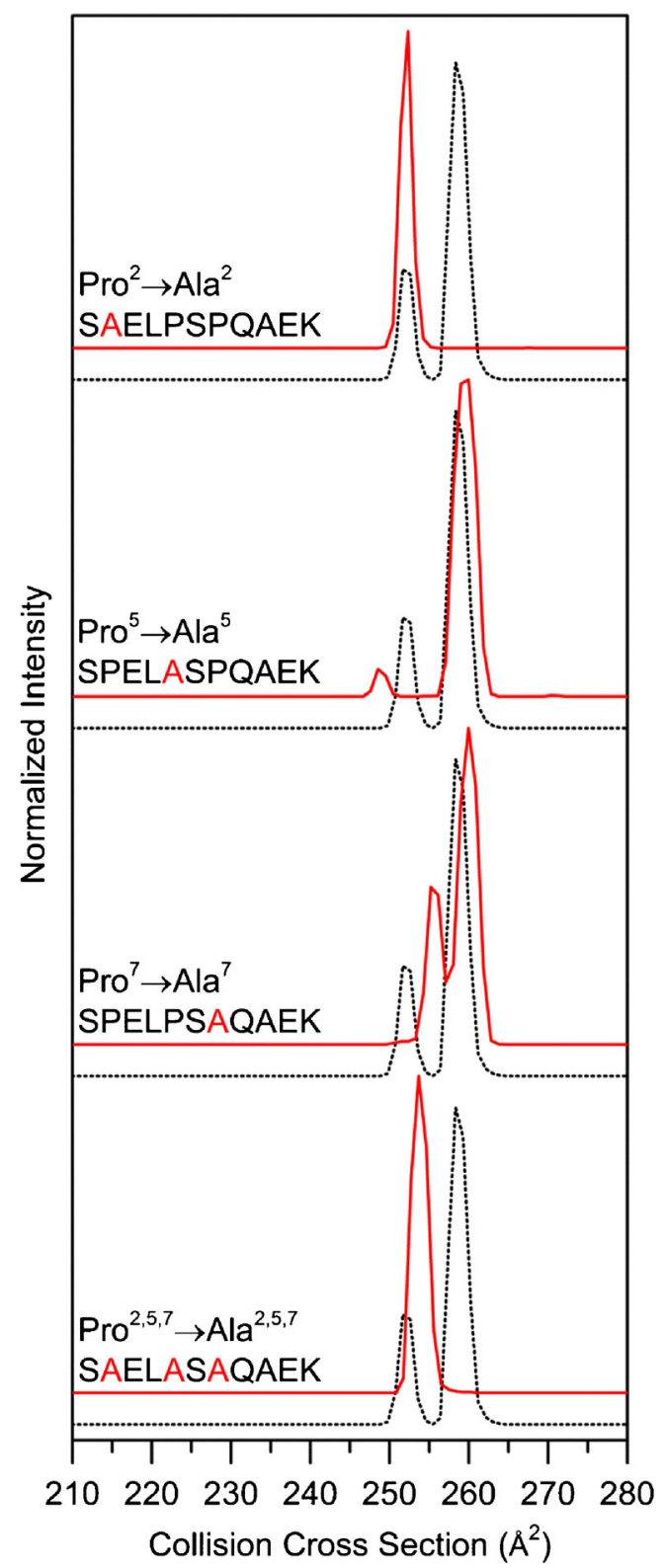

Figure 3. Collision cross section distributions for $\mathrm{Pro}^{2} \rightarrow \mathrm{Ala}$, $\mathrm{Pro}^{5} \rightarrow$ Ala, $\mathrm{Pro}^{7} \rightarrow$ Ala, and Pro ${ }^{2,5,7} \rightarrow$ Ala substituted peptides from the [SPELPSPQAEK $+2 \mathrm{H}]^{2+}$ ions. Substituted distributions are shown in red and the natural sequence is shown as a dotted trace for ease of comparison. Collision cross section distributions for substituted peptides are adjusted for the size difference between Ala and Pro as explained in text

$\mathrm{Ala}^{5}$ substituted peptide is $248 \AA^{2}$. The distribution for the $\mathrm{Ala}^{7}$ substituted peptide has two minor features at 251 and $255 \AA^{2}$. In addition to the difference in the number of peaks and their respective cross sections, the populations are also different. Several previous studies have focused on the local environment surrounding proline residues with significant attention to the identity of the residue $\mathrm{N}$-terminally adjacent to proline [40, 41]. A recent study suggests that the nonlocal environment influences cis-Pro residues in proteins found in the Protein Databank [42]. Our results suggest that distal residues influence the population of cis-trans isomers for peptide ions. One possible explanation for this is that intramolecular interactions that stabilize the structure of the peptide change upon substituting Ala for Pro. The backbone nitrogen atoms of proline residues are unable to participate in intramolecular $\mathrm{H}$ bonding because of the lack of hydrogen bond donor on the backbone nitrogen. However, Ala residues are able to form intramolecular H-bonds.

We have performed the triple substitution, Pro $^{2,5,7} \rightarrow$ Ala, as this peptide should represent the all trans peptide. The cross section distribution for the triply substituted peptide has a single peak located at $254 \AA^{2}$, less than $1 \%$ larger than the peak at $252 \AA^{2}$ for the peptide [SPELPSPQAEK $\left.+2 \mathrm{H}\right]^{2+}$ ion (Figure 3). Therefore, we have confirmed the conformer at 252 $\AA^{2}$ for the [SPELPSPQAEK $\left.+2 \mathrm{H}\right]^{2+}$ ion as the trans configuration at $\mathrm{Pro}^{2}, \mathrm{Pro}^{5}$, and $\mathrm{Pro}^{7}$ (Table 1).

\section{LPSPQAEK Pro $\rightarrow$ Ala Substitutions}

Figure 4 shows the collision cross section distribution for the Pro $\rightarrow$ Ala substituted peptides for the [LPSPQAEK $+2 \mathrm{H}]^{2+}$ ions. Upon $\mathrm{Pro}^{2} \rightarrow$ Ala substitution, we observe a distribution that is dominated by a single conformation at $215 \AA^{2}$. Although a minor feature is observed at $212 \AA^{2}$, it comprises less than $1 \%$ of the distribution. In contrast, the $\mathrm{Pro}^{4} \rightarrow$ Ala [LPSAQAEK + $2 \mathrm{H}]^{2+}$ distribution is strikingly similar to the [LPSPQAEK + $2 \mathrm{H}]^{2+}$ ion distribution, having two peaks at 213 and $216 \AA^{2}$ of nearly equal abundance. This suggests that the Pro $^{2}$ residue is important to observing multiple conformer families of nearly equal abundance. The conformer at $213 \AA^{2}$ is assigned as cis$\mathrm{Pro}^{2}$, trans-Pro ${ }^{4}$ (Table 1). Due to the presence of a peak $\sim 217$ $\AA^{2}$ for both the $\mathrm{Pro}^{2} \rightarrow$ Ala and $\mathrm{Pro}^{4} \rightarrow$ Ala distributions, this conformer is assigned as trans-Pro ${ }^{2}$ and trans-Pro ${ }^{4}$ (Table 1).

In order to confirm the cis-trans isomer assignment, we have analyzed the doubly substituted peptide $\mathrm{Pro}^{2,4} \rightarrow$ Ala. As shown in Figure 4, the cross section distribution for the doubly substituted $\mathrm{Pro}^{2,4} \rightarrow$ Ala peptide has a single peak at $217 \AA^{2}$. [LASAQAEK $\left.+2 \mathrm{H}\right]^{2+}$ likely represents the all-trans peptide. This provides further evidence that conformer at 217 $\AA^{2}$ for [LPSPQAEK $\left.+2 \mathrm{H}\right]^{2+}$ ions is trans-Pro ${ }^{2}$, trans-Pro ${ }^{4}$ (Table 1).

\section{SPQAEK Pro $\rightarrow$ Ala Substitutions}

Figure 5 shows the cross section distribution for the $\mathrm{Pro}^{2} \rightarrow$ Ala substituted peptide $[\mathrm{SAQAEK}+2 \mathrm{H}]^{2+}$. Upon substitution, the distribution has a single peak at $177 \AA^{2}$. This peak is aligned with the minor feature located at $178 \AA^{2}$ of the $[\text { SPQAEK }+2 \mathrm{H}]^{2+}$ distribution. Because this peptide contains a single Pro residue, the assignment is relatively straightforward. The Ser ${ }^{1}-\mathrm{Pro}^{2}$ peptide bond for the conformer at $178 \AA^{2}$ is assigned as trans because a similar conformer is observed for the $[\text { SAQAEK }+2 \mathrm{H}]^{2+}$, which likely has a trans-Ser ${ }^{1}-\mathrm{Ala}^{2}$ peptide bond. The conformer at $172 \AA^{2}$ is assigned as a cis-Pro ${ }^{2}$ peptide bond because it is not observed in the [SAQAEK + $2 \mathrm{H}]^{2+}$ distribution (Table 1). 
Table 1. Proline Cis-Trans Isomer Assignments

\begin{tabular}{lcclll}
\hline Sequence & Collision cross section $\left(\AA^{2}\right)$ & Normalized abundance $(\%)^{\mathrm{a}}$ & Pro $^{2}$ & Pro $^{4}$ & Pro $^{5}$ \\
\hline SPELPSPQAEK & 258 & 76.9 & Cis & - & Pro $^{7}$ \\
SPELPSPQAEK & 252 & 23.1 & Trans & - & Trans \\
LPSPQAEK & 213 & 57.0 & Cis & Trans & - \\
LPSPQAEK & 217 & 43.0 & Trans & Trans & - \\
SPQAEK & 172 & 83.4 & Cis & - & - \\
SPQAEK & 178 & 16.6 & Trans & - & - \\
\hline
\end{tabular}

${ }^{a}$ Peak abundances are the integrated area for each respective peak normalized to the total area for each collision cross section distribution shown in Figure 2.

\section{Examination of a Penultimate Proline-Containing Peptide Library}

In order to determine if the trend of peptides with a penultimate proline residue adopting multiple conformers is widely observed, ion mobility distributions were measured for 52 additional $[\mathrm{M}+2 \mathrm{H}]^{2+}$ peptides with a penultimate proline. IMSMS data was obtained from a library of synthesized peptides in a similar method to that shown in Figure 1, including both

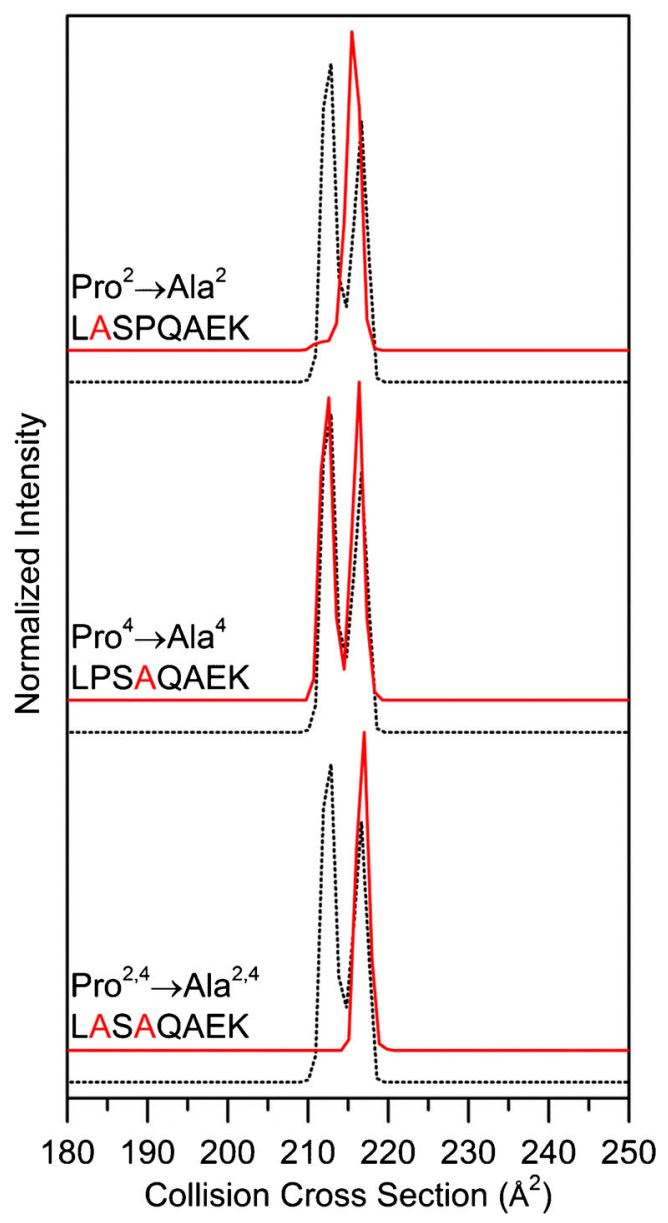

Figure 4. Collision cross section distributions for $\mathrm{Pro}^{2} \rightarrow \mathrm{Ala}$, $\mathrm{PrO}^{4} \rightarrow \mathrm{Ala}$, and $\mathrm{Pro}^{2,4} \rightarrow$ Ala substituted peptides from the [LPSPQAEK $+2 \mathrm{H}]^{2+}$ ions. Substituted distributions are shown in red and the natural sequence is shown as a dotted trace for ease of comparison. Collision cross section distributions for substituted peptides are adjusted for the size difference between Ala and Pro as explained in text truncated and full-length sequences. Figure 6 shows collision cross section distributions for several $[\mathrm{M}+2 \mathrm{H}]^{2+}$ peptides from this dataset. In both examples, we observe a single peak in the mobility distribution for the $\operatorname{Pro}^{1}$ peptide, multiple peaks of considerable abundance for the Pro ${ }^{2}$ peptides, and a distribution that is dominated by a single peak for the Pro $^{3}$ peptides.

If we consider all penultimate proline-containing peptides examined in this study, 46 of 58 (79\%) peptide sequences have multiple conformations in the IMS distribution. The complete list of sequences is listed in Supplementary Information (Table S1). Previous IMS-MS measurements by Counterman et al. found that $57 \%$ of proline-containing peptides had multiple features in the IMS-MS distribution [26]. It is worth mentioning that the previous measurement included $\mathrm{Pro}^{2}$ peptides. This suggests that penultimate proline-containing peptides adopt multiple conformations at a relatively high frequency across a range of peptide lengths and sequence compositions.

\section{Investigation into Cis- versus Trans-Stabilizing Intramolecular Interactions by Molecular Modeling}

Preliminary molecular modeling simulations were performed to provide insight into the differences in the intramolecular interactions that exist when Xaa-Pro bonds adopt the cis versus trans configuration. Simulations were performed on the [SPQAEK + $2 \mathrm{H}]^{2+}$ ion. This peptide was chosen because it has two well-

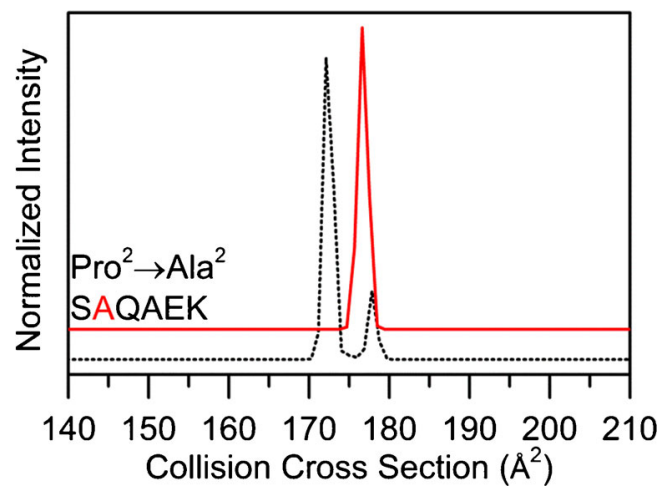

Figure 5. Collision cross section distributions for $\mathrm{Pro}^{2} \rightarrow$ Ala from the [SPQAEK $+2 \mathrm{H}]^{2+}$ ions. Substituted distributions are shown in red and the natural sequence is shown as a dotted trace for ease of comparison. Collision cross section distributions for substituted peptides are adjusted for the size difference between Ala and Pro as explained in text 


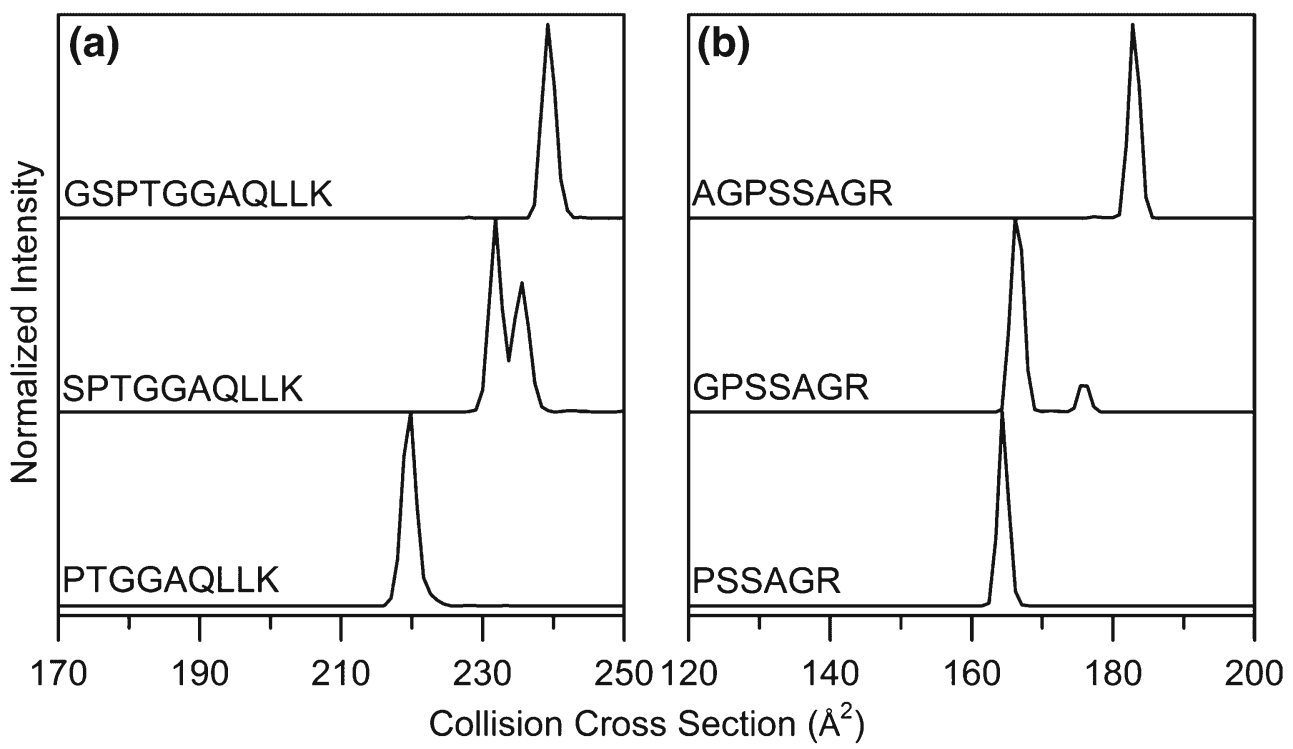

Figure 6. Collision cross section distributions for a series of $[\mathrm{M}+2 \mathrm{H}]^{2+}$ peptides containing Pro ${ }^{1}, \mathrm{Xaa}^{1}-\mathrm{Pro}^{2}$, and $\mathrm{Xaa}^{1}-\mathrm{Xaa}^{2}-\mathrm{Pro}^{3}$ sequences

resolved peaks in the cross section distribution, contains a single proline residue at position two, and is relatively small. Therefore, it provides an excellent model to probe the intramolecular interactions that stabilize both the cis and trans geometries of proline when located at the second position as shown in the experimental results.

Figure 7 shows two representative low-energy structures obtained from molecular modeling simulations. We selected the lowest energy structure for the cis-Pro isomer and a relatively low energy structure with similar deviation from the experimental results for the trans-Pro isomer. Collision cross section values are within $2.0 \%$ of the cross sections measured by IMS-MS; the modeled cis-Pro (Figure 7a) and trans-Pro (Figure $7 b$ ) conformers have calculated cross sections of 175 and $181 \AA^{2}$, respectively. Several general similarities exist between the two modeled structures - the Gln, Glu, and Lys side chains interact with the peptide backbone and the $\mathrm{N}$ - and C-terminus interact with each other. However, one key difference appears to be the disparity in the interactions with the oxygen atom of the second carbonyl group of the $\mathrm{Pro}^{2}$ residue. The cis-Pro peptide has an interaction between the protonated $\mathrm{N}$-terminus and the carbonyl oxygen of $\mathrm{Pro}^{2}$. Conversely, the trans-Pro peptide has an interaction between the protonated Lys side chain and the carbonyl oxygen of $\mathrm{Pro}^{2}$. Therefore, the interaction of the protonated N-terminus and the carbonyl of the $\mathrm{Pro}^{2}$ side chain may stabilize the cis geometry of the Ser ${ }^{1}$ $\mathrm{Pro}^{2}$ peptide bond. This may also explain why we did not observe similar distributions for conformations after an amino acid is added to the $\mathrm{N}$-terminus such that proline is in the third position. Upon addition of amino acids to the $\mathrm{N}$-terminus, this

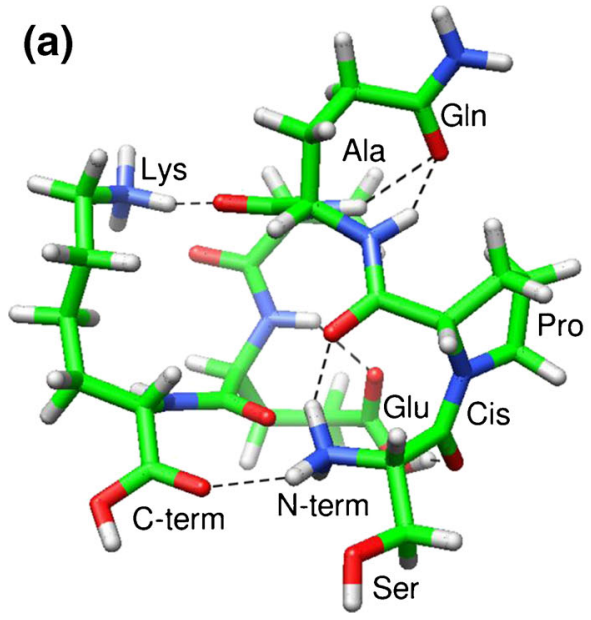

(b)

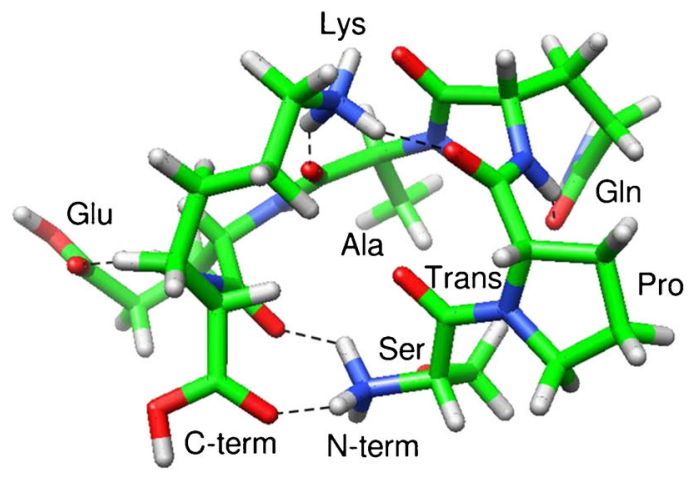

Figure 7. Representative structures of the $[\mathrm{SPQAEK}+2 \mathrm{H}]^{2+}$ ions with $\mathrm{Ser}^{1}-\mathrm{Pro}^{2}$ peptide bond in the cis (a) and trans (b) forms obtained from molecular modeling simulations. Amino acid side chains, terminal regions, and the geometry of the Ser ${ }^{1}-$ Pro $^{2}$ bond are labeled for clarity. $\mathrm{H}$-bonds determined using the recommended relaxed constrains in Chimera are represented as dotted lines [43] 
interaction may become less energetically favorable and a single conformation may become preferred.

\section{Simple Mechanistic Consideration}

In addition to the intramolecular interactions that are key to penultimate proline residues adopting multiple conformations mentioned above, it is worthwhile to consider potential mechanistic explanations for the preference of penultimate proline residues to adopt multiple conformations. One likely explanation is that the low steric impedance of rotating the $\mathrm{Xaa}^{1}-\mathrm{Pro}^{2}$ bond leads to a relatively low barrier between the cis and trans isomers. This allows the peptide to rotate more freely between the cis and trans isomers, making the tail region more flexible to adopt multiple conformers. For more internally located proline residues, the barrier to rotate larger regions of the peptide surrounding Xaa-Pro bonds may be increased compared with the terminal region. Also, intramolecular interactions surrounding the peptide may more effectively lock the Xaa-Pro peptide bond into a single isomer. We cannot rule out that a similar effect would be observed for proline residues located at the C-terminus. However, we have only studied tryptic peptides to date; therefore, all sequences analyzed terminate in Lys or Arg residues.

\section{Conclusions}

IMS-MS techniques and molecular modeling were used to examine the conformation families in a series of truncated and Pro $\rightarrow$ Ala substituted analogues of the peptide $[\text { SPELPSPQAEK }+2 \mathrm{H}]^{2+}$ ion. Our results are in good agreement with previous IMS-MS studies that suggest proline residues are important for multiple conformations [26, 27]. However, for this system, all proline residues are not equally important. We find a preference for $\mathrm{Pro}^{2}$ residues to adopt both the cis and trans isomer, resulting in multiple conformer families observed by IMS-MS. We find this trend to be true across a variety of peptide sequences; $\sim 80 \%$ of all penultimate proline-containing peptides analyzed have multiple stable conformations. This is not meant to imply that only penultimate proline residues can adopt multiple conformations; previous IMS-MS studies have observed Pro residues located at other positions in peptides with multiple conformations [26, 27]. Rather, we suggest that this position may have an intrinsic ability to stabilize both $c i s-$ and trans-Xaa ${ }^{1}-\mathrm{Pro}^{2}$ isomers. This is likely due to low steric impedance of the $\mathrm{N}$-terminal region, making this region of the peptide flexible and allowing for isomerization between the cis and trans forms of $\mathrm{Xaa}^{1}-\mathrm{Pro}^{2}$ peptide bonds.

Finally, it is interesting to consider the biological implications of Pro $^{2}$ establishing multiple conformations. Proline is commonly found in the second position in many biological systems, including neuro- and vasoactive peptides [2, 4]. Although the high frequency of Pro $^{2}$ residues is often hypothesized to stem from the ability of Pro residues to protect the Nterminal region from enzymatic degradation [1-3], it is possible that if $\mathrm{Xaa}^{1}-\mathrm{Pro}^{2}$ peptide bonds have a propensity to adopt both cis and trans isomers, this may have additional implications in peptide signaling.

\section{Acknowledgments}

The authors thank David Smiley and the DiMarchi research group at Indiana University for assistance with peptide synthesis. This work is supported by a grant from the NIH (R01 GM103725).

\section{References}

1. Falk, K., Rötzschke, O., Stevanović, S., Jung, G.: Rammensee, H-.G.: Pool sequencing of natural HLA-DR, DQ, and DP ligands reveals detailed peptide motifs, constraints of processing, and general rules. Immunogenetics 39, 230-242 (1994)

2. Vanhoof, G., Goossens, F., De Meester, I., Hendriks, D., Scharpé, S.: Proline motifs in peptides and their biological processing. FASEB J. 9, 736-744 (1995)

3. Nelson, C.A., Vidavsky, I., Viner, N.J., Gross, M.L., Unanue, E.R.: Amino-terminal trimming of peptides for presentation on major histocompatibility complex class II molecules. Proc. Natl. Acad. Sci. U. S. A. 94, 628-633 (1997)

4. Severini, C., Improta, G., Falconieri-Erspamer, G., Salvadori, S., Erspamer, V.: The tachykinin peptide family. Phamacol. Rev. 54, 285-322 (2002)

5. Sarkar, P., Reichman, C., Saleh, T., Birge, R.B., Kalodimos, C.G.: Proline cis-trans isomerization controls autoinhibition of a signaling protein. Mol. Cell 25, 413-426 (2007)

6. Brazin, K.N., Mallis, R.J., Fulton, D.B., Andreotti, A.H.: Regulation of the tyrosine kinase Itk by the peptidyl-prolyl isomerase cyclophilin A. Proc. Natl. Acad. Sci. U. S. A. 99, 1899-1904 (2002)

7. Lummis, S.C.R., Beene, D.L., Lee, L.W., Lester, H.A., Broadhurst, R.W., Dougherty, D.A.: Cis-trans isomerization at a proline opens the pore of a neurotransmitter-gated ion channel. Nature 438, 248-252 (2005)

8. Torbeev, V.Y., Hilvert, D.: Both the cis-trans equilibrium and isomerization dynamics of a single proline amide modulate B2-microglobulin amyloid assembly. Proc. Natl. Acad. Sci. U. S. A. 110, 20051-20056 (2013)

9. Stewart, D.E., Sarkar, A., Wampler, J.E.: Occurrence and role of cis peptide bonds in protein structures. J. Mol. Biol. 214, 253-260 (1990)

10. MacArthur, M.W., Thornton, J.M.: Influence of proline residues on protein conformation. J. Mol. Biol. 218, 397-412 (1991)

11. Andreotti, A.H.: Native state proline isomerization: an intrinsic molecular switch. Biochemistry 42, 9515-9524 (2003)

12. Fenn, J.B., Mann, M., Meng, C.K., Wong, S.F., Whitehouse, C.M.: Electrospray ionization for mass spectrometry of large biomolecules. Science 246, 64-71 (1989)

13. Clemmer, D.E., Jarrold, M.F.: Ion mobility measurements and their applications to clusters and biomolecules. J. Mass Spectrom. 32, 577-592 (1997)

14. Wyttenbach, T., Kemper, P.R., Bowers, M.T.: Design of a new electrospray ion mobility mass spectrometer. Int. J. Mass Spectrom. 212, 13-23 (2001)

15. McLean, J.A., Ruotolo, B.T., Gillig, K.J., Russell, D.H.: Ion mobility-mass spectrometry: a new paradigm for proteomics. Int. J. Mass Spectrom. 240, 301-315 (2005)

16. Bohrer, B.C., Merenbloom, S.I., Koeniger, S.L., Hilderbrand, A.E., Clemmer, D.E.: Biomolecule analysis by ion mobility spectrometry. Annu. Rev. Anal. Chem. 1, 293-327 (2008)

17. Kanu, A.B..., Dwivedi, P., Tam, M., Matz, L., Hill Jr., H.H.: Ion mobilitymass spectrometry. J. Mass Spectrom. 43, 1-22 (2008)

18. Ruotolo, B.T., Giles, K., Campuzano, I., Sandercock, A.M., Bateman, R.H., Robinson, C.V.: Evidence for macromolecular protein rings in the absence of bulk water. Science 310, 1658-1661 (2005)

19. Ruotolo, B.T., Robinson, C.V.: Aspects of native proteins are retained in vacuum. Curr. Opin. Chem. Biol. 10, 402-408 (2006)

20. Bleiholder, C., Dupuis, N.F., Wyttenbach, T., Bowers, M.T.: Ion mobilitymass spectrometry reveals a conformational conversion from random assembly to B-sheet in amyloid fibril formation. Nat. Chem. 3, 172-177 (2011) 
21. Pierson, N.A., Chen, L., Valentine, S.J., Russell, D.H., Clemmer, D.E.: Number of solution states of bradykinin from ion mobility and mass spectrometry measurements. J. Am. Chem. Soc. 133, 13810-13813 (2011)

22. Chen, L., Chen, S.-H., Russell, D.H.: An experimental study of the solventdependent self-assembly/disassembly and conformer preferences of gramicidin A. Anal. Chem. 85, 7826-7833 (2013)

23. Silveira, J.A., Fort, K.L., Kim, D., Servage, K.A., Pierson, N.A., Clemmer, D.E., Russell, D.H.: From solution to the gas phase: stepwise dehydration and kinetic trapping of Substance P reveals the origin of peptide conformations. J. Am. Chem. Soc. 135, 19147-19153 (2013)

24. Wyttenbach, T., Pierson, N.A., Clemmer, D.E., Bowers, M.T.: Ion mobility analysis of molecular dynamics. Annu. Rev. Phys. Chem. 65, 175-196 (2014)

25. Shi, L., Holliday, A.E., Shi, H., Zhu, F., Ewing, M.A., Russell, D.H., Clemmer, D.E.: Characterizing intermediates along the transition from polyproline I to polyproline II using ion mobility spectrometry-mass spectrometry. J. Am. Chem. Soc. 136, 12702-12711 (2014)

26. Counterman, A.E., Clemmer, D.E.: Cis-trans signatures of prolinecontaining tryptic peptides in the gas phase. Anal. Chem. 74, 1946-1951 (2002)

27. Pierson, N.A., Chen, L., Russell, D.H., Clemmer, D.E.: Cis-trans isomerizations of proline residues are key to bradykinin conformations. J. Am. Chem. Soc. 135, 3186-3192 (2013)

28. Schenk, E.R., Ridgeway, M.E., Park, M.A., Leng, F., Fernandez-Lima, F.: Isomerization kinetics of AT hook decapeptide solution structures. Anal. Chem. 86, 1210-1214 (2014)

29. Warnke, S., Baldauf, C., Bowers, M.T., Pagel, K., von Helden, G.: Photodissociation of conformer-selected ubiquitin ions reveals site-specific cis/ trans isomerization of proline peptide bonds. J. Am. Chem. Soc. 136, 10308-10314 (2014)

30. Wu, C.H., Yeh, L.-S.L., Huang, H., Arminski, L., Castro-Alvear, J., Chen, Y., Hu, Z., Kourtesis, P., Ledley, R.S., Suzek, B.E., Vinayaka, C.R., Zhang, J., Barker, W.C.: The protein information resource. Nucleic Acids Res. 31, 345-347 (2003)

31. Koeniger, S.L., Merenbloom, S.I., Valentine, S.J., Jarrold, M.F., Udseth, H.R., Smith, R.D., Clemmer, D.E.: An IMS-IMS analogue of MS-MS. Anal. Chem. 78, 4161-4174 (2006)
32. Merenbloom, S.I., Koeniger, S.L., Valentine, S.J., Plasencia, M.D. Clemmer, D.E.: IMS-IMS and IMS-IMS-IMS/MS for separating peptide and protein fragment ions. Anal. Chem. 78, 2802-2809 (2006)

33. Tang, K., Shvartsburg, A.A., Lee, H.-N., Prior, D.C., Buschbach, M.A., Li, F., Tolmachev, A.V., Anderson, G.A., Smith, R.D.: High-sensitivity ion mobility spectrometry/mass spectrometry using electrodynamic ion funnel interfaces. Anal. Chem. 77, 3330-3339 (2005)

34. Hoaglund, C.S., Valentine, S.J., Sporleder, C.R., Reilly, J.P., Clemmer, D.E.: Three-dimensional ion mobility/TOFMS analysis of electrosprayed biomolecules. Anal. Chem. 70, 2236-2242 (1998)

35. Mason, E.A., McDaniel, E.W.: Transport properties of ions in gases, pp. 129. Wiley, New York (1988)

36. Valentine, S.J., Counterman, A.E., Clemmer, D.E.: A database of 660 peptide ion cross sections: use of intrinsic size parameters for bona fide predictions of cross sections. J. Am. Soc. Mass Spectrom. 10, 1188-1211 (1999)

37. Srebalus-Barnes, C.A., Clemmer, D.E.: Assessing intrinsic side chain interactions between $\mathrm{i}$ and $\mathrm{i}+4$ residues in solvent-free peptides: a combinatorial gas-phase approach. J. Phys. Chem. A 107, 10566-10579 (2003)

38. Dilger, J.M., Valentine, S.J., Glover, M.S., Ewing, M.A., Clemmer, D.E.: A database of alkali metal-containing peptide cross sections: influence of metals on size parameters for specific amino acids. Int. J. Mass Spectrom. 330/332, 35-45 (2012)

39. Mesleh, M.F., Hunter, J.M., Shvartsburg, A.A., Schatz, G.C., Jarrold, M.F.: Structural information from ion mobility measurements: effects of the longrange potential. J. Phys. Chem. 100, 16082-16086 (1996)

40. Reimer, U., Scherer, G., Drewello, M., Kruber, S., Schutkowski, M., Fischer, G.: Side-chain effects on peptidyl-prolyl cis/trans isomerization. J. Mol. Biol. 279, 449-460 (1998)

41. Pal, D., Chakrabarti, P.: Cis peptide bonds in proteins: residues involved, their conformations, interactions, and locations. J. Mol. Biol. 294, 271-288 (1999)

42. Wathen, B., Jia, Z.: Local and nonlocal environments around cis peptides. J. Proteome Res. 7, 145-153 (2008)

43. Pettersen, E.F., Goddard, T.D., Huang, C.C., Couch, G.S., Greenblatt, D.M., Meng, E.C., Ferrin, T.E.: UCSF chimera-a visualization system for exploratory research and analysis. J. Comput. Chem. 25, 1605-1612 (2004) 